Abstract 569 Table 1 Headline data from the national lung cancer audit

\begin{tabular}{|c|c|c|c|c|c|c|c|c|c|}
\hline & 2005 & 2006 & 2007 & 2008 & 2009 & 2010 & 2011 & 2012 & 2013 \\
\hline \multicolumn{10}{|l|}{ Data Completeness } \\
\hline Number of cases & 10,920 & 16,922 & 20,639 & 25,757 & 30,158 & 30,329 & 31,429 & 31,003 & 30,508 \\
\hline PS & $66 \%$ & $77 \%$ & $80 \%$ & $87 \%$ & $88 \%$ & $84 \%$ & $89 \%$ & $91 \%$ & $93 \%$ \\
\hline Staging & $51 \%$ & $55 \%$ & $70 \%$ & $77 \%$ & $80 \%$ & $82 \%$ & $84 \%$ & $94 \%$ & $93 \%$ \\
\hline Treatment & $66 \%$ & $72 \%$ & $79 \%$ & $82 \%$ & $89 \%$ & $89 \%$ & $91 \%$ & $91 \%$ & $92 \%$ \\
\hline \multicolumn{10}{|l|}{ Process and Outcomes } \\
\hline HCR & $68 \%$ & $66 \%$ & $65 \%$ & $66.7 \%$ & $69.5 \%$ & $76.5 \%$ & $73.8 \%$ & $75.5 \%$ & $75.1 \%$ \\
\hline NSCLC NOS rate & - & $36 \%$ & $32 \%$ & $33.6 \%$ & $30 \%$ & $24 \%$ & $19 \%$ & $16 \%$ & $13 \%$ \\
\hline Discussed at MDT? & $79 \%$ & $84.3 \%$ & $86.8 \%$ & $88.6 \%$ & $93.2 \%$ & $96.1 \%$ & $95.9 \%$ & $95.6 \%$ & $95.2 \%$ \\
\hline Anti-cancer treatment? & $45 \%$ & $50 \%$ & $52 \%$ & $54 \%$ & $58.9 \%$ & $58.5 \%$ & $60.5 \%$ & $61.0 \%$ & $60.2 \%$ \\
\hline Overall resection rate & $9 \%$ & $9.4 \%$ & $10.3 \%$ & $11.2 \%$ & $13.9 \%$ & $13.9 \%$ & $15.3 \%$ & $15.5 \%$ & $15.4 \%$ \\
\hline NSCLC resection rate & $13.8 \%$ & $14.3 \%$ & $15.2 \%$ & $16 \%$ & $19 \%$ & $18.3 \%$ & $21 \%$ & $22 \%$ & $23 \%$ \\
\hline SCLC chemotherapy rate & $57.7 \%$ & $61.7 \%$ & $64.5 \%$ & $63 \%$ & $66 \%$ & $65 \%$ & $68 \%$ & $68 \%$ & $70 \%$ \\
\hline Seen by LCNS & - & - & - & $50.9 \%$ & $64.4 \%$ & $75.5 \%$ & $79.4 \%$ & $81.9 \%$ & $83.9 \%$ \\
\hline LCNS at diagnosis & - & - & - & $28.5 \%$ & $41 \%$ & $51.9 \%$ & $58.7 \%$ & $61.2 \%$ & $65.3 \%$ \\
\hline
\end{tabular}

audit as part of a new commissioning process, and the linkage with other developing datasets will allow the project to continue to realise the goal of improved and less variable outcomes and for patients with lung cancer.

\section{S70 RESULTS FROM THE FIRST NATIONAL LUNG CANCER ORGANISATIONAL AUDIT}

${ }^{1}$ IS Woolhouse, ${ }^{2} \mathrm{C}$ Meace, ${ }^{2} \mathrm{~K}$ Greenaway, ${ }^{1} \mathrm{P}$ Beckett, ${ }^{1} \mathrm{MD}$ Peake. ${ }^{1}$ Royal College of Physicians, London, UK; ${ }^{2}$ Health and Social Care Information Centre, Leeds, UK

\subsection{6/thoraxjnl-2014-206260.76}

Background National Lung Cancer Audit reports consistently demonstrate variation in diagnostic pathways, treatment rates and outcomes which are not wholly explained by case-mix. One possible explanation for this variation is different access to diagnostics and treatment specialists, however little is known about the provision of these services across England and Wales lung cancer services.

Methods An electronic survey was sent to all lung cancer lead clinicians in England and Wales in January 2014. The survey included seven questions for all MDTs on service provision, diagnostic services, staging services, and lung cancer treatment. There were a further 3 questions for treatment centres. Two reminders were sent and the survey closed in May 2014.

Results 128 records were submitted from 176 trusts. After removal of duplicate and empty records 101 were available for analysis. Mean (range) average number of patients discussed per MDT meeting is $26(5-88)$ and $29 \%$ Trusts have a separate diagnostic meeting. There is considerable variation in the mean (range) number of whole time equivalent (wte) on site lung cancer specialists e.g. thoracic pathologists $1.4(0-10)$, lung CNS $2.0(0.5-10)$ and respiratory physicians $3.9(0-20)$. Most diagnostic and staging procedures are available either on or off site, although medical thoracoscopy is not available at all to $14 \%$ Trusts. Chemotherapy, radiotherapy and surgery are available on site in $89 \%, 33 \%$ and $18 \%$ of Trusts, respectively. VAT lobectomy, stereotactic radiotherapy and radiofrequency ablation are not available at all to $6 \%, 5 \%$ and $10 \%$ of Trusts, respectively. Centres performing thoracic surgery report mean (range) wte number of surgeons at $1.5(0-6)$ and thoracic HDU beds at 4
(0-24). Acute oncology services are available to $92 \%$ chemotherapy treatment centres and $96 \%$ radiotherapy centres.

Conclusion The data provide a moderately representative snapshot of diagnostic and treatment services available for lung cancer patients in England and Wales. There is significant variation in the number of specialists available and some patients do not have access to key treatment modalities e.g. VAT lobectomy. Further work is required to determine how this relates to patient experience and outcomes. All Trusts are encouraged to submit validated data for the next round of organisational audit.

\section{S71 ARE QUALITY STANDARDS AND ACCREDITED CENTRES FOR MEDIASTINAL STAGING WITH EBUS NEEDED? A REPORT FROM THE MANCHESTER CANCER EBUS GROUP}

${ }^{1} M$ Evison, ${ }^{1} P$ Crosbie, ${ }^{1} \mathrm{~J}$ Morris, ${ }^{1} \mathrm{~J}$ Martin, ${ }^{1} \mathrm{R}$ Shah, ${ }^{1} \mathrm{H}$ Doran, ${ }^{2} \mathrm{~J}$ Hoyle, ${ }^{3} \mathrm{~S}$ Bailey, ${ }^{3} \mathrm{D}$ Rana, ${ }^{4} \mathrm{R}$ Sundar, ${ }^{1} \mathrm{R}$ Booton. ${ }^{1}$ University Hospital South Manchester, Manchester, UK; ${ }^{2}$ Peninne Acute NHS Trust, Manchester, UK; ${ }^{3}$ Central Manchester NHS Foundation Trust, Manchester, UK; ${ }^{4}$ Wrightington, Wigan and Leigh NHS Foundation Trust, Wigan, UK

\subsection{6/thoraxjnl-2014-206260.77}

Introduction Mediastinal staging in lung cancer is a core function of EBUS-TBNA. There has been an explosion of EBUS -TBNA services across the UK over recent years. However, quality standards and adherence to such standards are not widely known. The aim of this study was to describe the current practices of four independent EBUS centres serving a large UK cancer network.

Materials and Methods In 2012, the number of centres providing EBUS-TBNA in this Network increased from one to four. This prompted the development of an EBUS sub-group and service specification that mandates the collection of pre-defined data for all EBUS procedures. Analysis of this prospectively maintained database was undertaken for this report.

Results 741 lung cancer patients underwent EBUS-TBNA in the study period. $56.4 \%(418 / 741)$ were for nodal staging, with the remaining performed for pathological confirmation of lung cancer. The proportion of staging procedures performed at each centre varied significantly (range $4.8 \%-80.3 \%, \mathrm{p}<0.0001$ ). In those patients undergoing EBUS for mediastinal staging, the average number of lymph stations sampled per procedure varied from 1.3 to 1.9 across the four centres and the proportion of 